\title{
Robust Document Retrieval and Individual Evidence Modeling for Fact Extraction and Verification.
}

\author{
Tuhin Chakrabarty and Tariq Alhindi \\ Department of Computer Science \\ Columbia University \\ tc2896@columbia.edu \\ tariq@es.columbia.edu
}

\author{
Smaranda Muresan \\ Department of Computer Science \\ Data Science Institute \\ Columbia University \\ smara@columbia.edu
}

\begin{abstract}
This paper presents the ColumbiaNLP submission for the FEVER Workshop Shared Task. Our system is an end-to-end pipeline that extracts factual evidence from Wikipedia and infers a decision about the truthfulness of the claim based on the extracted evidence. Our pipeline achieves significant improvement over the baseline for all the components (Document Retrieval, Sentence Selection and Textual Entailment) both on the development set and the test set. Our team finished 6th out of 24 teams on the leader-board based on the preliminary results with a FEVER score of 49.06 on the blind test set compared to 27.45 of the baseline system.
\end{abstract}

\section{Introduction and Background}

Fact checking is a type of investigative journalism where experts examine the claims published by others for their veracity. The claims can range from statements made by public figures to stories reported by other publishers. The end goal of a fact checking system is to provide a verdict on whether the claim is true, false, or mixed. Several organizations such as FactCheck . org and Politifact are devoted to such activities.

The FEVER Shared task aims to evaluate the ability of a system to verify information using evidence from Wikipedia. Given a claim involving one or more entities (mapping to Wikipedia pages), the system must extract textual evidence (sets of sentences from Wikipedia pages) that supports or refutes the claim and then using this evidence, it must label the claim as Supported, Refuted or NotEnoughInfo. The dataset for the shared task was introduced by Thorne et al. (2018) and consists of 185,445 claims. Table 1 shows three instances from the data set with the claim, the evidence and the verdict.

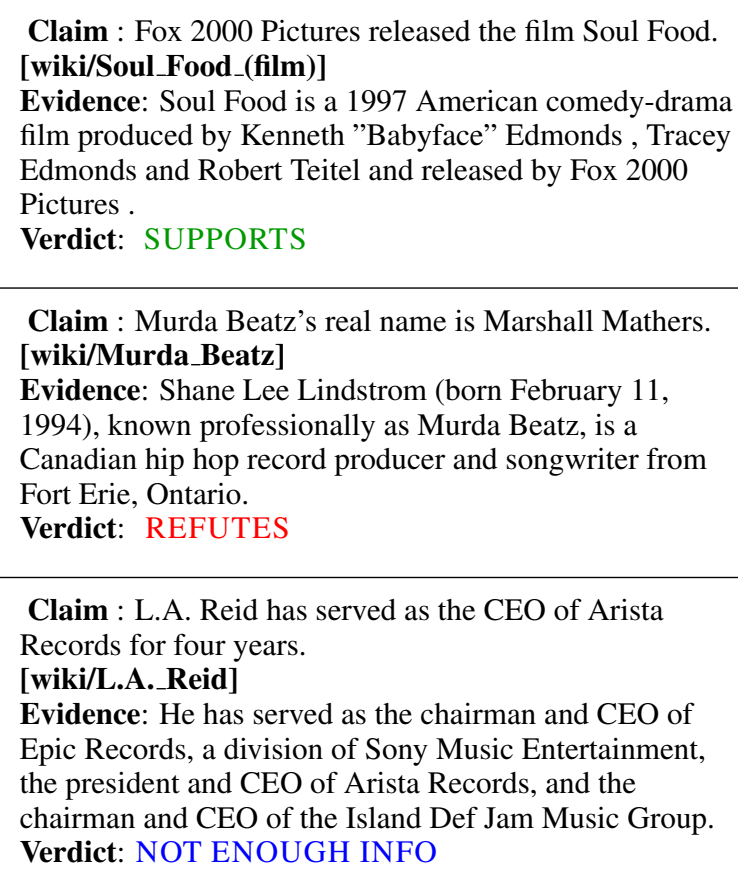

Claim : Murda Beatz's real name is Marshall Mathers. [wiki/Murda_Beatz]

Evidence: Shane Lee Lindstrom (born February 11, 1994), known professionally as Murda Beatz, is a

Canadian hip hop record producer and songwriter from

Fort Erie, Ontario.

Verdict: REFUTES

Claim : L.A. Reid has served as the CEO of Arista Records for four years.

[wiki/L.A._Reid]

Evidence: He has served as the chairman and CEO of Epic Records, a division of Sony Music Entertainment, the president and CEO of Arista Records, and the chairman and CEO of the Island Def Jam Music Group. Verdict: NOT ENOUGH INFO

Table 1: Examples of claims, the extracted evidence from Wikipedia and the verdicts from the shared task dataset (Thorne et al., 2018)

The baseline system described by Thorne et al. (2018) uses 3 major components:

- Document Retrieval: Given a claim, identify relevant documents from Wikipedia which contain the evidence to verify the claim. Thorne et al. (2018) used the document retrieval component from the DrQA system (Chen et al., 2017), which returns the $\mathrm{k}$ nearest documents for a query using cosine similarity between binned unigram and bigram TF-IDF vectors.

- Sentence Selection: Given the set of retrieved document, identify the candidate evidence sentences. Thorne et al. (2018) used a modified document retrieval component of 
DrQA (Chen et al., 2017) to select the top most similar sentences w.r.t the claim, using bigram TF-IDF with binning.

- Textual Entailment: For the entailment task, training is done using labeled claims paired with evidence (labels are SUPPORTS, REFUTES, NOT ENOUGH INFO). Thorne et al. (2018) used the decomposable attention model (Parikh et al., 2016) for this task. For the case where multiple sentences are required as evidence, the strings were concatenated.

Our system implements changes in all three modules (Section 2), which leads to significant improvements both on the development and test sets. On the shared task development set our document retrieval approach covers $94.4 \%$ of the claims requiring evidence, compared to $55.30 \%$ in the baseline. Further, on the dev set our evidence recall is improved by 33 points over the baseline. For entailment, our model improves the baseline by 7.5 points on dev set. Overall, our end-to-end system shows an improvement of 19.56 in FEVER score compared to the baseline (50.83 vs. 31.27 ) on the dev set. On the blind test set we achieve an evidence recall of 75.89 and an entailment accuracy of 57.45 (9 points above baseline) resulting in a FEVER score of 49.06 (Section 3). Together with the results we discuss some lessons learned based on our error analysis and release our code ${ }^{1}$.

\section{Methods}

\subsection{Document Retrieval}

Document Retrieval is a crucial step when building an end-to-end system for fact extraction and verification. Missing a relevant document could lead to missed evidence, while non-relevant documents would add noise for the subsequent tasks of sentence selection and textual entailment. We propose a multi-step approach for retrieving documents relevant to the claims.

- Google Custom Search API: Wang et al. (2018) looked at retrieving relevant documents for fact-checking articles, looking at generating candidates via search. Inspired by this, we first use the Custom Search API of Google to retrieve documents having information about the claim. We add the token

\footnotetext{
${ }^{1}$ https://github.com/tuhinjubcse/FEVER-EMNLP
}

wikipedia to the claim and issue a query and collect the top 2 results.

- Named Entity Recognition: Second, we use the AllenNLP (Gardner et al., 2017) pretrained bidirectional language model (Peters et al., 2017) for named entity recognition ${ }^{2}$. After finding the named entities in the claim, we use Wikipedia python API ${ }^{3}$ to collect the top wikipedia document returned by the API for each named entity.

- Dependency Parse: Third, to increase the chance of detecting relevant entities in the claim, we find the first lower case verb phrase (VP) in the dependency parse tree and query the Wikipedia API with all the tokens before the VP. The reason for emphasizing lower case verb phrase is to avoid missing entities in claims such as "Finding Dory was directed by X", where the relevant entity is "Finding Dory".

To deal with entity ambiguity, we also add the token film in our query where the claim contains keywords such as film, stars, premiered and directed by. For example in "Marnie was directed by Whoopi Goldberg.", Marnie can refer to both wikipedia pages Marnie (film) and Marnie. Our point of interest here is Marnie (film). We only experimented with $\mathrm{film}$ to capture the performance gains. One of our future goals is to build better computational models to handle entity ambiguity or entity linking.

- Combined: We use the union of the documents returned by the three approaches as the final set of relevant documents to be used by the Sentence Selection module.

\begin{tabular}{|l|c|c|}
\hline Method & Avg k & Coverage \\
\hline Google API & 2 & $79.5 \%$ \\
\hline NER & 2 & $77.1 \%$ \\
\hline Dependency Parse & 1 & $80.0 \%$ \\
\hline Combined & 3 & $\mathbf{9 4 . 4 \%}$ \\
\hline \hline (Thorne et al., 2018) & 5 & $55.3 \%$ \\
\hline
\end{tabular}

Table 2: Coverage of claims that can be fully supported or refuted by the retrieved documents (dev set)

Table 2 shows the percentage of claims that can be fully supported or refuted by the retrieved docu-

\footnotetext{
${ }^{2}$ http://demo.allennlp.org/named-entity-recognition

${ }^{3}$ https://pypi.org/project/wikipedia/
} 
ments before sentence selection on the dev set. We see that our best approach (combined) achieved a high coverage $94.4 \%$ compared to the baseline (Thorne et al., 2018) of 55.3\%. Because we do not have the gold evidences for the blind test set we cannot report the claim coverage using our pipeline .

\subsection{Sentence Selection}

For sentence selection, we used the modified document retrieval component of DrQA (Chen et al., 2017) to select sentences using bigram TF-IDF with binning as proposed by (Thorne et al., 2018). We extract the top 5 most similar sentences from the k-most relevant documents using the TF-IDF vector similarity. Our evidence recall is 78.4 as compared to 45.05 in the development set of FEVER (Thorne et al., 2018), which demonstrates the importance of document retrieval in fact extraction and verification. On the blind test set our sentence selection approach achieves an evidence recall of 75.89 .

However, even though TF-IDF proves to be a strong baseline for sentence selection we noticed on the dev set that using all 5 evidences together introduced additional noise to the entailment model. To solve this, we further filtered the top 3 evidences from the selected 5 evidences using distributed semantic representations. Peters et al. (2018) show how deep contextualized word representations model both complex characteristics of word use (e.g., syntax and semantics), and usage across various linguistic contexts. Thus, we used the ELMo embeddings to convert the claim and evidence to vectors. We then calculated cosine similarity between claim and evidence vectors and extracted the top 3 sentences based on the score. Because there was no penalty involved for poor evidence precision, we returned all five selected sentences as our predicted evidence but used only the top three sentences for the entailment model.

\subsection{Textual Entailment}

The final stage of our pipeline is recognizing textual entailment. Unlike Thorne et al. (2018), we did not concatenate evidences, but trained our model for each claim-evidence pair. For recognizing textual entailment we used the model introduced by Conneau et al. (2017) in their work on supervised learning of universal sentence representations.

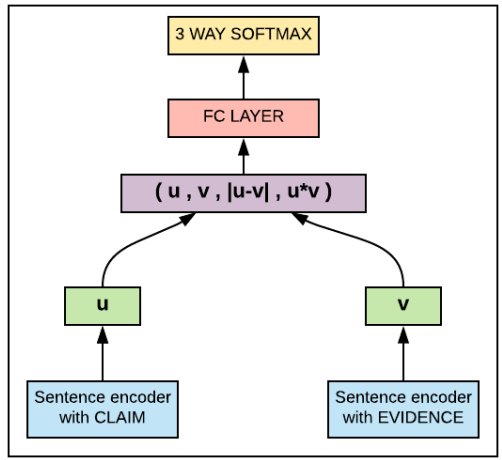

Figure 1: The architecture for recognizing textual entailment (adapted from (Conneau et al., 2017))

The architecture is presented in Figure 1. We use bidirectional LSTMs (Hochreiter and Schmidhuber, 1997) with max-pooling to encode the claim and the evidence. The text encoder provides dense feature representation of an input claim or evidence. Formally, for a sequence of $\mathrm{T}$ words $w_{t=1, . ., T}$, the BiLSTM layer generates a sequence of $h_{t}$ vectors, where $h_{t}$ is the concatenation of a forward and a backward LSTM output. The hidden vectors $h_{t}$ are then converted into a single vector using max-pooling, which chooses the maximum value over each dimension of the hidden units. Overall, the text encoder can be treated as an operator Text $\rightarrow R^{d}$ that provides d dimensional encoding for a given text.

Out of vocabulary issues in pre-trained word embeddings are a major bottleneck for sentence representations. To solve this we use fastText embeddings (Bojanowski et al., 2017) which rely on subword information. Also, these embeddings were trained on Wikipedia corpus making them an ideal choice for this task.

As shown in Figure 1, the shared sentence encoder outputs a representation for the claim $u$ and the evidence $v$. Once the sentence vectors are generated, the following three methods are applied to extract relations between the claim and the evidence: (i) concatenation of the two representations (u, v); (ii) element-wise product $\mathrm{u}^{*} \mathrm{v}$ and (iii) absolute element-wise difference $|u-v|$. The resulting vector, which captures information from both the claim and the evidence, is fed into a 3-class classifier consisting of fully connected layers culminating in a softmax layer.

For the final class label, we experimented first by taking the majority prediction of the three 
(claim, evidence) pairs as our entailment label but this led to lower accuracy on the dev set. So our final predictions are based on the rule outlined in the Algorithm 1, where SUPPORTS $=S$, REFUTES $=R$, NOT ENOUGH INFO $=N$ and $C$ is a count function. Because the selected evidences were inherently noisy and our pipeline did not concatenate evidences together we chose this rule over majority prediction to mitigate the dominance of prediction of NOT ENOUGH INFO class.

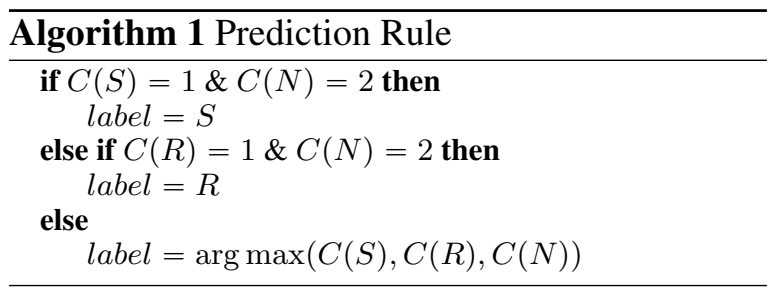

We also experimented by training a classifier which takes confidence scores of all the three claim evidence pairs along with their position in the document and trained a boosted tree classifier but the accuracy did not improve. Empirically the rule gave us the best results on the dev set and thus used it to obtain the final label.

Table 3 shows the 3 way classification accuracy using the textual entailment model described above.

\begin{tabular}{|c|c|}
\hline DataSet & Accuracy \\
\hline Shared Task Dev & 58.77 \\
\hline Blind Test Set & 57.45 \\
\hline
\end{tabular}

Table 3: 3 way classification results

Our entailment accuracy on the shared task dev and test set is 7 and 9 points better than the baseline respectively.

Implementation Details. The batch size is kept as 64. The model is trained for 15 epochs using Adam optimizer with a learning rate of 0.001 . The size of the LSTM hidden units is set to 512 and for the classifier, we use a MLP with 1 hidden-layer of 512 hidden units. The embedding dimension of the words is set to 300 .

\section{End to End Results and Error Analysis}

Table 4 shows the overall FEVER score obtained by our pipeline on the dev and test sets. In the provisional ranking our system ranked 6th.

On closer investigation we find that neither TFIDF nor sentence embedding based approaches are

\begin{tabular}{|c|c|c|}
\hline Data & Pipeline & FEVER \\
\hline \multirow{2}{*}{ DEV } & (Thorne et al., 2018) & 31.27 \\
\cline { 2 - 3 } & Ours & $\mathbf{5 0 . 8 3}$ \\
\hline \multirow{2}{*}{ TEST } & (Thorne et al., 2018) & 27.45 \\
\cline { 2 - 3 } & Ours & $\mathbf{4 9 . 0 6}$ \\
\hline
\end{tabular}

Table 4: FEVER scores on shared task dev and test set

perfect when it comes to sentence selection, although TF-IDF works better.

\begin{tabular}{|l|l|}
\hline Fox 2000 Pictures released the film & \multirow{2}{*}{0.29} \\
Soul Food & \\
Soul Food is a 1997 American comedy-drama & \\
film produced by Kenneth & \\
"Babyface" Edmonds, Tracey & \\
Edmonds and Robert Teitel and released by & \\
Fox 2000 Pictures & \\
\hline
\end{tabular}

Table 5: Cosine similarity between claim and supporting evidence

Table 5 goes on to prove that we cannot rely on models that entirely depend on semantics. In spite of the two sentences being similar, the cosine similarity between them is poor mostly because the evidence contains a lot of extra information which might not be relevant to the claim and difficult for the model to understand.

\begin{tabular}{l} 
At seventeen or eighteen years of age, he joined Plato's \\
Academy in Athens and remained there until the age of \\
thirty-seven (c. 347 BC) \\
\hline $\begin{array}{l}\text { Shortly after Plato died, Aristotle left Athens and at the } \\
\text { request of Philip II of Macedon,tutored Alexander the } \\
\text { Great beginning in } 343 \text { BC }\end{array}$ \\
\hline
\end{tabular}

Table 6: The top evidence is selected by Annotators and the bottom evidence by our pipeline

We also found instances where the predicted evidence is correct but it does not match the gold evidence. For the claim "Aristotle spent time in Athens", both evidences given in Table 6 support it, but still our system gets penalized on not being able to match the gold evidence.

We found quite a few annotations to be incorrect and hence the FEVER scores are lower than expected. Table 7 show two instances where the gold labels for the claims was NOT ENOUGH INFO, while in fact they should have been SUPPORTS and REFUTES, respectively.

Table 8 reflects the fact that NOT ENOUGH INFO is often hard to predict and that is where our model needs to improve more.

The lines between SUPPORTS and NOT ENOUGH INFO are often blurred as shown in 
Claim: Natural Born Killers was directed by Oliver Stone Evidence: Natural Born Killers is a 1994 American satirical crime film directed by Oliver Stone and starring Woody Harrelson, Juliette Lewis , Robert Downey Jr. , Tom Sizemore , and Tommy Lee Jones .

Claim:Anne Rice was born in New Jersey

Evidence: Born in New Orleans, Rice spent much of her early life there before moving to Texas, and later to San Francisco

Table 7: Wrong gold label (NOT ENOUGH INFO)

\begin{tabular}{|c|c|c|c|}
\hline & $\mathrm{S}$ & $\mathrm{N}$ & $\mathrm{R}$ \\
\hline $\mathrm{S}$ & $\mathbf{4 6 3 5}$ & 1345 & 686 \\
\hline $\mathrm{N}$ & 2211 & $\mathbf{3 2 6 9}$ & 1186 \\
\hline $\mathrm{R}$ & 1348 & 1470 & $\mathbf{3 8 4 8}$ \\
\hline
\end{tabular}

Table 8: Confusion matrix of entailment predictions on shared task dev set

Table 8. Our models need better understanding of semantics to be able to identify these. Table 9 shows one such example where the gospel keyword becomes the discriminative factor.

Claim: Happiness in Slavery is a gospel song

by Nine Inch Nails

Evidence: Happiness in Slavery,is a song by American industrial rock band Nine Inch Nails from their debut extended play (EP), Broken(1992)

Table 9: Example where our model predicts SUPPORTS for a claim labeled as NOT ENOUGH INFO

\section{Conclusion}

The FEVER shared task is challenging primarily because the annotation requires substantial manual effort. We presented an end-to-end pipeline to automate the human effort and showed empirically that our model outperforms the baseline by a large margin. We also provided a thorough error analysis which highlights some of the shortcomings of our models and potentially of the gold annotations.

\section{References}

Piotr Bojanowski, Edouard Grave, Armand Joulin, and Tomas Mikolov. 2017. Enriching word vectors with subword information. Transactions of the Association for Computational Linguistics, 5:135-146.

Danqi Chen, Adam Fisch, Jason Weston, and Antoine Bordes. 2017. Reading wikipedia to answer opendomain questions. pages 1870-1879. In Proceedings of the 55th Annual Meeting of the Association for Computational Linguistics (Volume 1: Long Papers).

Alexis Conneau, Douwe Kiela, Holger Schwenk, Loïc Barrault, and Antoine Bordes. 2017. Supervised learning of universal sentence representations from natural language inference data. In Proceedings of the 2017 Conference on Empirical Methods in Natural Language Processing, pages 670-680, Copenhagen, Denmark. Association for Computational Linguistics.

Matt Gardner, Joel Grus, Oyvind Tafjord Mark Neumann, Pradeep Dasigi, Nelson Liu, Matthew Peters, Michael Schmitz, and Luke Zettlemoyer. 2017. Allennlp: A deep semantic natural language processing platform.

Sepp Hochreiter and Jurgen Schmidhuber. 1997. Long short-term memory. In Neural computation, 791, pages $1735-1780$.

Ankur Parikh, Dipanjan Das Oscar Tackstr om, and Jakob Uszkoreit. 2016. A decomposable attention model for natural language inference. pages 22492255. In Proceedings of the 2016 Conference on Empirical Methods in Natural Language Processing. Association for Computational Linguistics, Austin, Texas.

Matthew E. Peters, Waleed Ammar, Chandra Bhagavatula, and Russell Power. 2017. Semi-supervised sequence tagging with bidirectional language models. In $A C L$.

Matthew E. Peters, Mark Neumann, Mohit Iyyer, Matt Gardner, Christopher Clark, Kenton Lee, and Luke Zettlemoyer. 2018. Deep contextualized word representations. In Proc. of NAACL.

James Thorne, Andreas Vlachos, Christos Christodoulopoulos, and Arpit Mittal. 2018. Fever: a large-scale dataset for fact extraction and verification. pages 809-819. Proceedings of NAACL-HLT 2018.

Xuezhi Wang, Cong Yu, Simon Baumgartner, and Flip Korn. 2018. Relevant document discovery for factchecking articles. pages 525-533. WWW' 18 Companion Proceedings of the The Web Conference 2018. 